DOI: https://doi.org/10.24297/jam.v20i.8952

\title{
Squared prime numbers
}

Methods giving all prime numbers endless

Dr Gunnar Appelqvist

\section{Abstract;}

The prime numbers are the building numbers of the number series. They are dividable only with themselves and 1. These prime numbers build all numbers in the number series. The number 89 is a prime while 87 is a composite number containing the primes 3 and 29. The number 87 therefore is not a prime number because it contains two primes. The prime numbers occur quite close in the number series even if they eventually slightly thin. Euclid, though, proved $300^{\text {th }} \mathrm{BC}$ that there is an infinite number of primes. The highest prime number known right now is $2^{82} 589933-1$. I have discovered a formula giving all prime numbers endless. This as a result from other prime constructions I newly found showing how different prime constellations refer to each other in one or several squares. Finally, I have discovered another method giving all prime numbers endless, also explaining why they occur as they do.

\section{Nomenclature}

The word prime square, origin square or, for example, 11-square, 17-square (or any prime) denote a square created from a certain prime number, the so-called origin prime.

The origin rectangle is the rectangle formed when the right vertical line of the prime square is left out.

The word corner square is the largest possible square drawn from each corner without overlapping each other.

The word starting prime refer to the prime numbers $5,7,11,13,17$ and 19 when used to find all primes endless.

\section{Construction}

Let us start with constructing a square with as many boxes, squared of course, as the prime number you start with. The Microsoft Excel should be suitable to use. If we use the origin prime number 7, the empty square looks like this:

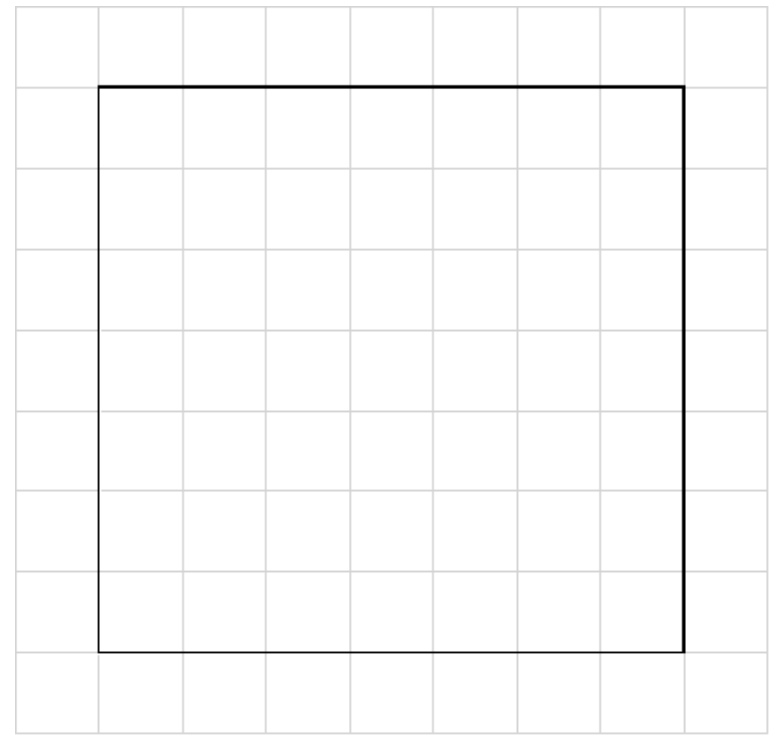

We then continue to go through the number series line by line. As soon as a prime number turns up, we note that number in the box. After having gone through all 49 boxes in the square the primes are placed like this: 


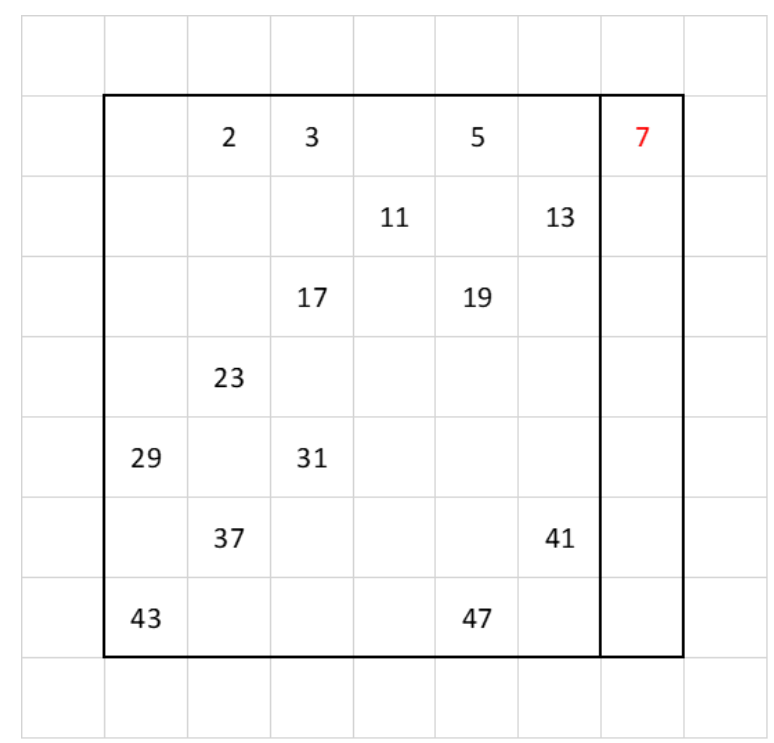

We continue to survey the square, box by box, starting on the upper left box. Every new prime number is noted in the same way. During the third survey a collision with an occupied box appears. The prime number 101 should have been noted in a box already occupied by 3 . We then have to create a new square and place 101 in that very same box in this second square (or just leave the number, if we intend to use just the first square). Thereafter we continue to note the primes in the first square until another collision appears.

Even in the second square collisions may appear, which in turn demand a third square. But after that we go on with the first square until this one is filled with prime numbers. Some gaps then remain in the second square and we continue according to the same principles until the three first squares derived from the origin prime 7 are filled. These three squares, noted A, B and C, look like this:

\section{Square 1}

\begin{tabular}{|c|c|c|c|c|c|c|c|c|c|}
\hline A & & & & & & & & & $: 7=$ \\
\hline & 197 & 2 & 3 & 53 & 5 & 251 & 7 & 511 & 73 \\
\hline & 449 & 107 & 59 & 11 & 61 & 13 & & 700 & 100 \\
\hline & 113 & 163 & 17 & 67 & 19 & 167 & & 546 & 78 \\
\hline & 71 & 23 & 73 & 613 & 173 & 223 & & 1176 & 168 \\
\hline & 29 & 79 & 31 & 179 & 131 & 83 & & 532 & 76 \\
\hline & 281 & 37 & 283 & 137 & 89 & 41 & & 868 & 124 \\
\hline & 43 & 191 & 241 & 193 & 47 & 97 & & 812 & 116 \\
\hline & 1183 & 602 & 707 & 1253 & 525 & 875 & & & \\
\hline$: 7=$ & 169 & 86 & 101 & 179 & 75 & 125 & & & \\
\hline & $\sum$ & 5145 & : & $7^{2}$ & $=$ & 105 & & & \\
\hline
\end{tabular}




\section{Square 2}

\begin{tabular}{|c|c|c|c|c|c|c|c|c|c|}
\hline B & & & & & & & & & $: 7=$ \\
\hline & 491 & 149 & 101 & 151 & 103 & 349 & 7 & 1344 & 192 \\
\hline & 547 & 401 & 157 & 109 & 257 & 307 & & 1778 & 254 \\
\hline & 211 & 359 & 311 & 263 & 313 & 461 & & 1918 & 274 \\
\hline & 659 & 317 & 269 & 809 & 271 & 419 & & 2744 & 392 \\
\hline & 127 & 373 & 227 & 277 & 229 & 181 & & 1414 & 202 \\
\hline & 379 & 233 & 479 & 431 & 383 & 139 & & 2044 & 292 \\
\hline & 239 & 877 & 829 & 389 & 439 & 293 & & 3066 & 438 \\
\hline & 2653 & 2709 & 2373 & 2429 & 1995 & 2149 & & & \\
\hline$: 7=$ & 379 & 387 & 339 & 347 & 285 & 307 & & & \\
\hline & $\Sigma$ & 14308 & : & $7^{2}$ & $=$ & 292 & & & \\
\hline
\end{tabular}

\section{Square 3}

\begin{tabular}{|c|c|c|c|c|c|c|c|c|c|}
\hline C & & & & & & & & & $: 7=$ \\
\hline & 883 & 443 & 199 & 347 & 397 & 643 & 7 & 2912 & 416 \\
\hline & 743 & 499 & 353 & 599 & 1237 & 503 & & 3934 & 562 \\
\hline & 701 & 457 & 409 & 557 & 509 & 853 & & 3486 & 498 \\
\hline & 463 & 709 & 367 & 907 & 467 & 811 & & 3724 & 532 \\
\hline & 421 & 569 & 521 & 571 & 523 & 769 & & 3374 & 482 \\
\hline & 673 & 331 & 577 & 823 & 677 & 433 & & 3514 & 502 \\
\hline & 337 & 1171 & 1123 & 487 & 733 & 587 & & 4438 & 634 \\
\hline & 4221 & 4179 & 3549 & 4291 & 4543 & 4599 & & & \\
\hline$: 7=$ & 603 & 597 & 507 & 613 & 649 & 657 & & & \\
\hline & $\Sigma$ & 25382 & : & $7^{2}$ & $=$ & 518 & & & \\
\hline
\end{tabular}

It should be emphasized that the right vertical line, starting with the origin prime ( 7 in this case) does not participate in the investigation more than filling up the square with prime numbers.

The sum of the primes, line by line, horizontal as well as vertical, is divisible with the origin prime itself ( 7 in this case). This is shown by the figures alongside or underneath every line in the upper example. The total sum of the square's numbers is shown at the bottom after the sum sign $\sum$. This total sum always is evenly divisible with the origin prime squared $\left(7^{2}\right.$ in this case).

Below I present the results regarding the two first squares ( $A$ and $B$ ) concerning the origin prime 11 , and the first square $(A)$ concerning the origin prime 17. The quotients after the sum of every line are left out in the 17square. 


\begin{tabular}{|c|c|c|c|c|c|c|c|c|c|c|c|c|c|}
\hline A & & & & & & & & & & & & & $: 11=$ \\
\hline & 727 & 2 & 3 & 367 & 5 & 127 & 7 & 613 & 251 & 131 & 11 & 2233 & 203 \\
\hline & 617 & 13 & 619 & 257 & 137 & 17 & 139 & 19 & 383 & 263 & & 2464 & 224 \\
\hline & 23 & 1597 & 509 & 389 & 269 & 149 & 29 & 151 & 31 & 1847 & & 4994 & 454 \\
\hline & 397 & 277 & 157 & 37 & 401 & 281 & 887 & 41 & 163 & 43 & & 2684 & 244 \\
\hline & 1013 & 167 & 47 & 653 & 2711 & 1381 & 293 & 173 & 53 & 659 & & 7150 & 650 \\
\hline & 419 & 541 & 179 & 59 & 181 & 61 & 1151 & 547 & 911 & 307 & & 4356 & 396 \\
\hline & 67 & 431 & 311 & 191 & 71 & 193 & 73 & 1163 & 317 & 197 & & 3014 & 274 \\
\hline & 199 & 79 & 443 & 1049 & 929 & 83 & 1657 & 569 & 449 & 571 & & 6028 & 548 \\
\hline & 89 & 211 & 1301 & 1181 & 577 & 457 & 337 & 701 & 97 & 461 & & 5412 & 492 \\
\hline & 463 & 101 & 223 & 103 & 467 & 347 & 227 & 107 & 229 & 109 & & 2376 & 216 \\
\hline & 353 & 233 & 113 & 719 & 599 & 479 & 359 & 239 & 1087 & 241 & & 4422 & 402 \\
\hline & 4367 & 3652 & 3905 & 5005 & 6347 & 3575 & 5159 & 4323 & 3971 & 4829 & & & \\
\hline \multirow[t]{2}{*}{$: 11=$} & 397 & 332 & 355 & 455 & 577 & 325 & 469 & 393 & 361 & 439 & & & \\
\hline & $\Sigma$ & 45133 & : & $11^{2}$ & $=$ & 373 & & & & & & & \\
\hline
\end{tabular}

\begin{tabular}{|c|c|c|c|c|c|c|c|c|c|c|c|c|c|}
\hline \multirow[t]{2}{*}{ B } & & & & & & & & & & & & & $: 11=$ \\
\hline & 1453 & 607 & 487 & 1093 & 1699 & 853 & 491 & 1097 & 977 & 373 & 11 & 9130 & 830 \\
\hline & 859 & 739 & 1103 & 499 & 379 & 743 & 2801 & 503 & 1109 & 1231 & & 9966 & 906 \\
\hline & 991 & 2081 & 751 & 631 & 1237 & 1117 & 271 & 877 & 757 & 2089 & & 10802 & 982 \\
\hline & 881 & 761 & 641 & 521 & 643 & 523 & 1129 & 283 & 647 & 769 & & 6798 & 618 \\
\hline & 2707 & 409 & 773 & 1621 & 2953 & 2591 & 1019 & 1867 & 1021 & 1627 & & 16588 & 1508 \\
\hline & 661 & 1993 & 421 & 1511 & 907 & 787 & 1877 & 1031 & 1153 & 1033 & & 11374 & 1034 \\
\hline & 1277 & 673 & 1279 & 433 & 313 & 677 & 557 & 1889 & 2011 & 439 & & 9548 & 868 \\
\hline & 683 & 563 & 2137 & 1291 & 1171 & 809 & 2141 & 811 & 691 & 1297 & & 11594 & 1054 \\
\hline & 331 & 937 & 1543 & 1423 & 1061 & 941 & 821 & 1427 & 823 & 1187 & & 10494 & 954 \\
\hline & 947 & 827 & 1433 & 587 & 709 & 2767 & 953 & 349 & 1439 & 593 & & 10604 & 964 \\
\hline & 1321 & 1201 & 839 & 2897 & 1567 & 1447 & 601 & 1933 & 1571 & 967 & & 14344 & 1304 \\
\hline & 12111 & 10791 & 11407 & 12507 & 12639 & 13255 & 12661 & 12067 & 12199 & 11605 & & & \\
\hline$: 11=$ & 1101 & 981 & 1037 & 1137 & 1149 & 1205 & 1151 & 1097 & 1109 & 1055 & & & \\
\hline & $\Sigma$ & 121242 & : & $11^{2}$ & $=$ & 1002 & & & & & & & \\
\hline
\end{tabular}




\begin{tabular}{|c|c|c|c|c|c|c|c|c|c|c|c|c|c|c|c|c|c|c|}
\hline A & A & B & C & D & E & $\mathrm{F}$ & G & H & 1 & $\mathrm{~J}$ & K & L & $M$ & $N$ & O & $\mathrm{P}$ & $\mathrm{Q}$ & \\
\hline 1 & 3469 & 2 & 3 & 293 & 5 & 1451 & 7 & 1453 & 587 & 877 & 11 & 3191 & 13 & 881 & 593 & 883 & 17 & 13719 \\
\hline 2 & 307 & 19 & 887 & 599 & 311 & 23 & 313 & 1181 & 1471 & 2339 & 317 & 29 & 2053 & 31 & 2633 & 4079 & & 16592 \\
\hline 3 & 613 & 1481 & 37 & 1483 & 617 & 907 & 41 & 331 & 43 & 911 & 1201 & 2069 & 47 & 337 & 1783 & 3229 & & 15130 \\
\hline 4 & 919 & 53 & 1499 & 1789 & 2657 & 1213 & 347 & 59 & 349 & 61 & 929 & 641 & 353 & 643 & 1511 & 67 & & 13090 \\
\hline 5 & 647 & 359 & 71 & 3251 & 73 & 941 & 653 & 2099 & 1811 & 367 & 79 & 947 & 659 & 2683 & 83 & 373 & & 15096 \\
\hline 6 & 953 & 2399 & 2111 & 89 & 379 & 3559 & 2693 & 1249 & 383 & 673 & 3853 & 97 & 1543 & 677 & 389 & 101 & & 21148 \\
\hline 7 & 103 & 971 & 683 & 2129 & 107 & 397 & 109 & 977 & 2423 & 401 & 113 & 1559 & 3583 & 983 & 5897 & 2141 & & 22576 \\
\hline 8 & 409 & 1277 & 1567 & 701 & 991 & 2437 & 1571 & 127 & 2729 & 2441 & 419 & 131 & 421 & 1289 & 1579 & 1291 & & 19380 \\
\hline 9 & 137 & 1583 & 139 & 2741 & 719 & 431 & 1877 & 433 & 1301 & 1013 & 1303 & 2749 & 149 & 439 & 151 & 1019 & & 16184 \\
\hline 10 & 443 & 733 & 1601 & 157 & 4493 & 3049 & 449 & 739 & 1607 & 163 & 1031 & 743 & 1033 & 167 & 457 & 3637 & & 20502 \\
\hline 11 & 1327 & 461 & 173 & 463 & 3643 & 1621 & 3067 & 467 & 179 & 2203 & 181 & 1049 & 761 & 1051 & 4231 & 3943 & & 24820 \\
\hline 12 & 2789 & 3079 & 479 & 191 & 1637 & 193 & 1061 & 773 & 1063 & 197 & 487 & 199 & 2801 & 5981 & 491 & 3671 & & 25092 \\
\hline 13 & 1361 & 5119 & 4253 & 6277 & 787 & 499 & 211 & 1657 & 4259 & 503 & 1949 & 2239 & 1373 & 1663 & 797 & 509 & & 33456 \\
\hline 14 & 1667 & 223 & 1091 & 1381 & 1093 & 227 & 2251 & 229 & 1097 & 809 & 521 & 233 & 523 & 5437 & 1103 & 2549 & & 20434 \\
\hline 15 & 239 & 5153 & 241 & 1109 & 821 & 2267 & 823 & 2269 & 3137 & 1693 & 827 & 1117 & 251 & 541 & 1409 & 1699 & & 23596 \\
\hline 16 & 1123 & 257 & 547 & 1993 & 2861 & 839 & 1129 & 263 & 1709 & 1999 & 5179 & 1423 & 557 & 269 & 2293 & 271 & & 22712 \\
\hline 17 & 1429 & 563 & 853 & 1721 & 277 & 1723 & 857 & 569 & 281 & 571 & 283 & 1151 & 863 & 1153 & 6067 & 577 & & 18938 \\
\hline & 17935 & 23732 & 16235 & 26367 & 21471 & 21777 & 17459 & 14875 & 24429 & 17221 & 18683 & 19567 & 16983 & 24225 & 31467 & 30039 & & \\
\hline & $\Sigma$ & 342465 & : & $17^{2}$ & $=$ & 1185 & & & & & & & & & & & & \\
\hline
\end{tabular}

\section{Reflections}

The corner squares are noted a, b, c, and d clockwise. The upper left corner (a) and lower right corner (c) in the origin square (or upper right and lower left) do reflect each other. The reflections come clear when you find that the sum is evenly divisible with the origin prime squared. This also goes for two different reflections $(a, c$ and $b$, d), and even if added with reflections in the middle horizontal line. Three examples are showed below. The 7square's third square $(C)$, the 11-square's second square $(B)$ and the 17-square's first square $(A)$. The corner square's denotations are used. 


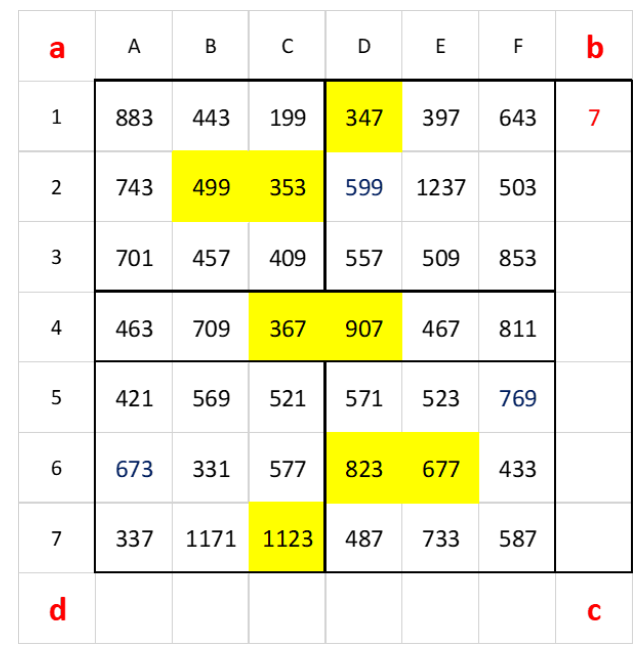

The three reflection's sum ( $a, c$ and b, $d$ as well as the middle horizontal line) is 5096 which divided with $7^{2}$ gives 104.

\begin{tabular}{|c|c|c|c|c|c|c|c|c|c|c|c|}
\hline a & A & B & C & D & E & $\mathrm{F}$ & G & $\mathrm{H}$ & 1 & J & b \\
\hline 1 & 1453 & 607 & 487 & 1093 & 1699 & 853 & 491 & 1097 & 977 & 373 & 11 \\
\hline 2 & 859 & 739 & 1103 & 499 & 379 & 743 & 2801 & 503 & 1109 & 1231 & \\
\hline 3 & 991 & 2081 & 751 & 631 & 1237 & 1117 & 271 & 877 & 757 & 2089 & \\
\hline 4 & 881 & 761 & 641 & 521 & 643 & 523 & 1129 & 283 & 647 & 769 & \\
\hline 5 & 2707 & 409 & 773 & 1621 & 2953 & 2591 & 1019 & 1867 & 1021 & 1627 & \\
\hline 6 & 661 & 1993 & 421 & 1511 & 907 & 787 & 1877 & 1031 & 1153 & 1033 & \\
\hline 7 & 1277 & 673 & 1279 & 433 & 313 & 677 & 557 & 1889 & 2011 & 439 & \\
\hline 8 & 683 & 563 & 2137 & 1291 & 1171 & 809 & 2141 & 811 & 691 & 1297 & \\
\hline 9 & 331 & 937 & 1543 & 1423 & 1061 & 941 & 821 & 1427 & 823 & 1187 & \\
\hline 10 & 947 & 827 & 1433 & 587 & 709 & 2767 & 953 & 349 & 1439 & 593 & \\
\hline 11 & 1321 & 1201 & 839 & 2897 & 1567 & 1447 & 601 & 1933 & 1571 & 967 & \\
\hline d & & & & & & & & & & & C \\
\hline
\end{tabular}

The three reflection's sum ( $a, c$ and $b, d$ as well as the middle horizontal line) is 12584 which divided with $11^{2}$ gives 104. 


\begin{tabular}{|c|c|c|c|c|c|c|c|c|c|c|c|c|c|c|c|c|c|}
\hline $\mathbf{a}$ & A & B & C & D & E & $\mathrm{F}$ & G & H & 1 & $\mathrm{~J}$ & K & L & M & $\mathrm{N}$ & 0 & $\mathrm{P}$ & b \\
\hline 1 & 3469 & 2 & 3 & 293 & 5 & 1451 & 7 & 1453 & 587 & 877 & 11 & 3191 & 13 & 881 & 593 & 883 & 17 \\
\hline 2 & 307 & 19 & 887 & 599 & 311 & 23 & 313 & 1181 & 1471 & 2339 & 317 & 29 & 2053 & 31 & 2633 & 4079 & \\
\hline 3 & 613 & 1481 & 37 & 1483 & 617 & 907 & 41 & 331 & 43 & 911 & 1201 & 2069 & 47 & 337 & 1783 & 3229 & \\
\hline 4 & 919 & 53 & 1499 & 1789 & 2657 & 1213 & 347 & 59 & 349 & 61 & 929 & 641 & 353 & 643 & 1511 & 67 & \\
\hline 5 & 647 & 359 & 71 & 3251 & 73 & 941 & 653 & 2099 & 1811 & 367 & 79 & 947 & 659 & 2683 & 83 & 373 & \\
\hline 6 & 953 & 2399 & 2111 & 89 & 379 & 3559 & 2693 & 1249 & 383 & 673 & 3853 & 97 & 1543 & 677 & 389 & 101 & \\
\hline 7 & 103 & 971 & 683 & 2129 & 107 & 397 & 109 & 977 & 2423 & 401 & 113 & 1559 & 3583 & 983 & 5897 & 2141 & \\
\hline 8 & 409 & 1277 & 1567 & 701 & 991 & 2437 & 1571 & 127 & 2729 & 2441 & 419 & 131 & 421 & 1289 & 1579 & 1291 & \\
\hline 9 & 137 & 1583 & 139 & 2741 & 719 & 431 & 1877 & 433 & 1301 & 1013 & 1303 & 2749 & 149 & 439 & 151 & 1019 & \\
\hline 10 & 443 & 733 & 1601 & 157 & 4493 & 3049 & 449 & 739 & 1607 & 163 & 1031 & 743 & 1033 & 167 & 457 & 3637 & \\
\hline 11 & 1327 & 461 & 173 & 463 & 3643 & 1621 & 3067 & 467 & 179 & 2203 & 181 & 1049 & 761 & 1051 & 4231 & 3943 & \\
\hline 12 & 2789 & 3079 & 479 & 191 & 1637 & 193 & 1061 & 773 & 1063 & 197 & 487 & 199 & 2801 & 5981 & 491 & 3671 & \\
\hline 13 & 1361 & 5119 & 4253 & 6277 & 787 & 499 & 211 & 1657 & 4259 & 503 & 1949 & 2239 & 1373 & 1663 & 797 & 509 & \\
\hline 14 & 1667 & 223 & 1091 & 1381 & 1093 & 227 & 2251 & 229 & 1097 & 809 & 521 & 233 & 523 & 5437 & 1103 & 2549 & \\
\hline 15 & 239 & 5153 & 241 & 1109 & 821 & 2267 & 823 & 2269 & 3137 & 1693 & 827 & 1117 & 251 & 541 & 1409 & 1699 & \\
\hline 16 & 1123 & 257 & 547 & 1993 & 2861 & 839 & 1129 & 263 & 1709 & 1999 & 5179 & 1423 & 557 & 269 & 2293 & 271 & \\
\hline 17 & 1429 & 563 & 853 & 1721 & 277 & 1723 & 857 & 569 & 281 & 571 & 283 & 1151 & 863 & 1153 & 6067 & 577 & \\
\hline d & & & & & & & & & & & & & & & & & C \\
\hline
\end{tabular}

The two reflection's sum ( $a, c$ and b, d) is 24276 which divided with $17^{2}$ gives 84 .

These reflections also bring that the circumferences, from start box to start box, are evenly divisible with the origin prime squared. The 17-square below clarify this by showing the different circumferences in different colors. Their sums, as well as the quotients after division with $17^{2}$, are presented beneath. 


\begin{tabular}{|c|c|c|c|c|c|c|c|c|c|c|c|c|c|c|c|c|c|c|c|c|}
\hline A & A & B & c & D & E & $\mathrm{F}$ & G & $\mathrm{H}$ & I & J & K & L & M & $N$ & 0 & $\mathrm{P}$ & & & & \\
\hline 1 & 3469 & 2 & 3 & 293 & 5 & 1451 & 7 & 1453 & 587 & 877 & 11 & 3191 & 13 & 881 & 593 & 883 & 17 & 74273 & $: 17^{2}$ & 257 \\
\hline 2 & 307 & 19 & 887 & 599 & 311 & 23 & 313 & 1181 & 1471 & 2339 & 317 & 29 & 2053 & 31 & 2633 & 4079 & & & & \\
\hline 3 & 613 & 1481 & 37 & 1483 & 617 & 907 & 41 & 331 & 43 & 911 & 1201 & 2069 & 47 & 337 & 1783 & 3229 & & 76296 & $: 17^{2}$ & 264 \\
\hline 4 & 919 & 53 & 1499 & 1789 & 2657 & 1213 & 347 & 59 & 349 & 61 & 929 & 641 & 353 & 643 & 1511 & 67 & & & & \\
\hline 5 & 647 & 359 & 71 & 3251 & 73 & 941 & 653 & 2099 & 1811 & 367 & 79 & 947 & 659 & 2683 & 83 & 373 & & 57800 & $: 17^{2}$ & 200 \\
\hline 6 & 953 & 2399 & 2111 & 89 & 379 & 3559 & 2693 & 1249 & 383 & 673 & 3853 & 97 & 1543 & 677 & 389 & 101 & & & & \\
\hline 7 & 103 & 971 & 683 & 2129 & 107 & 397 & 109 & 977 & 2423 & 401 & 113 & 1559 & 3583 & 983 & 5897 & 2141 & & 45084 & $: 17^{2}$ & 156 \\
\hline 8 & 409 & 1277 & 1567 & 701 & 991 & 2437 & 1571 & 127 & 2729 & 2441 & 419 & 131 & 421 & 1289 & 1579 & 1291 & & & & \\
\hline 9 & 137 & 1583 & 139 & 2741 & 719 & 431 & 1877 & 433 & 1301 & 1013 & 1303 & 2749 & 149 & 439 & 151 & 1019 & & 37570 & $: 17^{2}$ & 130 \\
\hline 10 & 443 & 733 & 1601 & 157 & 4493 & 3049 & 449 & 739 & 1607 & 163 & 1031 & 743 & 1033 & 167 & 457 & 3637 & & & & \\
\hline 11 & 1327 & 461 & 173 & 463 & 3643 & 1621 & 3067 & 467 & 179 & 2203 & 181 & 1049 & 761 & 1051 & 4231 & 3943 & & 27166 & $: 17^{2}$ & 94 \\
\hline 12 & 2789 & 3079 & 479 & 191 & 1637 & 193 & 1061 & 773 & 1063 & 197 & 487 & 199 & 2801 & 5981 & 491 & 3671 & & & & \\
\hline 13 & 1361 & 5119 & 4253 & 6277 & 787 & 499 & 211 & 1657 & 4259 & 503 & 1949 & 2239 & 1373 & 1663 & 797 & 509 & & 17340 & $: 17^{2}$ & 60 \\
\hline 14 & 1667 & 223 & 1091 & 1381 & 1093 & 227 & 2251 & 229 & 1097 & 809 & 521 & 233 & 523 & 5437 & 1103 & 2549 & & & & \\
\hline 15 & 239 & 5153 & 241 & 1109 & 821 & 2267 & 823 & 2269 & 3137 & 1693 & 827 & 1117 & 251 & 541 & 1409 & 1699 & & 6936 & $: 17^{2}$ & 24 \\
\hline 16 & 1123 & 257 & 547 & 1993 & 2861 & 839 & 1129 & 263 & 1709 & 1999 & 5179 & 1423 & 557 & 269 & 2293 & 271 & & & & \\
\hline 17 & 1429 & 563 & 853 & 1721 & 277 & 1723 & 857 & 569 & 281 & 571 & 283 & 1151 & 863 & 1153 & 6067 & 577 & & & & \\
\hline & & & & & & & & & & & & & & & & & & & & \\
\hline
\end{tabular}

You may summarize the presentation above concerning the reflections inside a prime square in the following way.

You start with a square out of an optional prime number. I have above showed squares derived from the primes 7, 11 and 17.

You count de boxes in the first square (A) starting on the first box. Every time a prime number appears you right it down in that box. When a box is occupied by a former prime you either leave it out or transfer it to the same box in a second square (B).

When a square is filled with primes you subdivide it into four corner squares, as big as possible, denoted a, b, $c$ and $d$ clockwise. You also get a center line between the left and right vertical sides.

Irrespective of what kind of constellation you activate this is what you find:

1. Every constellation in the corner square a and/or $d$ added to a corresponding constellation in the corner square $b$ and/or $c$ is evenly divisible with the origin prime.

2. Every constellation in the corner square $a$ and/or $b$ added to a corresponding constellation in the corner square $d$ and/or $c$ is not evenly divisible with the origin prime.

3. Every reflecting constellation inside two of the opposed diagonal corner squares, possibly summarized with any optional reflecting constellation inside the two other diagonal corner squares, is evenly divisible with the origin prime squared. You may even add a reflection inside the center line and get this result.

This explains, among other things, why every circumference in the colored example above (origin square 17) is evenly divisible with $17^{2}$. Every half line is reflecting the opposite diagonal side, as well as you may reckon with the reflection inside the center line. 
In this way the prime numbers in the origin square are reflecting each other in different ways. The term "reflection" indicate that the sum is divisible with the origin prime, either plain or squared.

The prime numbers cooperating in different ways are identic with the prime series up to when the collisions are turning up, and thereafter the primes do not follow any particular pattern. The primes in the 7-square are spread out as below (number 7 is not marked).

\begin{tabular}{|c|c|c|c|c|c|c|c|c|c|c|c|}
\hline & & & & & & & & & & \\
\hline
\end{tabular}

\section{A formula giving every prime number without end}

How, then, is the relationship between the first prime square $(A)$ and the second one (B)? In the second square the prime numbers are mostly higher than in the first one, and if you compare a specific box the prime in the second square is always higher than in the first square. The box number one in the 7-square is 197 while in the second square it is 491 . The difference between 491 and 197 is 294. The interesting thing is that 294 is evenly divisible with $7^{2} \times 2=98$ which gives $298 / 98=3$. Every prime in the second square has got a similar relation to its corresponding number in the first square. For instance, $439-47=392$ which give us $392 / 98=4$. In this way every prime number in the second 7 -square is related to the corresponding prime number in the first square by adding a few number of $7^{2} \times 2$ to the prime number in the first square. My conjecture is that this is valid concerning all prime squares.

Now let us start with the lowest applicable prime number 3 and its square $3^{2}$. Double it and you get 18 . We add it to the six next starting prime numbers $5,7,11,13,17$ and 19. Let us start with the number 5 . After a few adds you get a prime, and after another few adds you get another higher one. In this way you continue as long as you want to. Thereafter you go further with the other numbers $(7,11,13,17$ and 19$)$ in the same way. Every starting prime in the examples below has got its own color, facilitating to follow the procedure. In this way every prime number is detected, and the prime series is complete as far as we choose to go on.

\section{A formula giving all prime numbers is:}

$$
\begin{aligned}
& 5+18 \times n,+18 \times n,+18 \times n \ldots \text { without end } \\
& 7+18 \times n,+18 \times n,+18 \times n \ldots \text { without end } \\
& 11+18 \times n,+18 \times n,+18 \times n \ldots \text { without end } \\
& 13+18 \times n,+18 \times n,+18 \times n \ldots \text { without end } \\
& 17+18 \times n,+18 \times n,+18 \times n \ldots \text { without end } \\
& 19+18 \times n,+18 \times n,+18 \times n \text {... without end }
\end{aligned}
$$

The letter $n$ in the formula stands for how many 18-adds you have to do before the next prime is found.

I will now illustrate this in three examples. The first one begins with the starting primes in the formula $(5,7,11$, $13,17,19)$ up to the prime number 1889 . The second example starts from the prime number 95783 . The color marks are shown in the first example and show the connection to the starting prime. The third illustration is 
fetched from a quite high section in the prime number series starting from the prime 888888888888811 . Even in this case all the detected primes are drawn from the starting primes. Wherever you start in the prime series there is a connection to the starting primes. My investigations, so far, show the same unequivocal result, which strengthens the evidence of this formula.

\begin{tabular}{|c|c|c|c|c|c|c|c|c|c|}
\hline 2 & 3 & 5 & 7 & 11 & 13 & 17 & 19 & 23 & 29 \\
\hline 31 & 37 & 41 & 43 & 47 & 53 & 59 & 61 & 67 & 71 \\
\hline 73 & 79 & 83 & 89 & 97 & 101 & 103 & 107 & 109 & 113 \\
\hline 127 & 131 & 137 & 139 & 149 & 151 & 157 & 163 & 167 & 173 \\
\hline 179 & 181 & 191 & 193 & 197 & 199 & 211 & 223 & 227 & 229 \\
\hline 233 & 239 & 241 & 251 & 257 & 263 & 269 & 271 & 277 & 281 \\
\hline 283 & 293 & 307 & 311 & 313 & 317 & 331 & 337 & 347 & 349 \\
\hline 353 & 359 & 367 & 373 & 379 & 383 & 389 & 397 & 401 & 409 \\
\hline 419 & 421 & 431 & 433 & 439 & 443 & 449 & 457 & 461 & 463 \\
\hline 467 & 479 & 487 & 491 & 499 & 503 & 509 & 521 & 523 & 541 \\
\hline 547 & 557 & 563 & 569 & 571 & 577 & 587 & 593 & 599 & 601 \\
\hline 607 & 613 & 617 & 619 & 631 & 641 & 643 & 647 & 653 & 659 \\
\hline 661 & 673 & 677 & 683 & 691 & 701 & 709 & 719 & 727 & 733 \\
\hline 739 & 743 & 751 & 757 & 761 & 769 & 773 & 787 & 797 & 809 \\
\hline 811 & 821 & 823 & 827 & 829 & 839 & 853 & 857 & 859 & 863 \\
\hline 877 & 881 & 883 & 887 & 907 & 911 & 919 & 929 & 937 & 941 \\
\hline 947 & 953 & 967 & 971 & 977 & 983 & 991 & 997 & 1009 & 1013 \\
\hline 1019 & 1021 & 1031 & 1033 & 1039 & 1049 & 1051 & 1061 & 1063 & 1069 \\
\hline 1087 & 1091 & 1093 & 1097 & 1103 & 1109 & 1117 & 1123 & 1129 & 1151 \\
\hline 1153 & 1163 & 1171 & 1181 & 1187 & 1193 & 1201 & 1213 & 1217 & 1223 \\
\hline 1229 & 1231 & 1237 & 1249 & 1259 & 1277 & 1279 & 1283 & 1289 & 1291 \\
\hline 1297 & 1301 & 1303 & 1307 & 1319 & 1321 & 1327 & 1361 & 1367 & 1373 \\
\hline 1381 & 1399 & 1409 & 1423 & 1427 & 1429 & 1433 & 1439 & 1447 & 1451 \\
\hline 1453 & 1459 & 1471 & 1481 & 1483 & 1487 & 1489 & 1493 & 1499 & 1511 \\
\hline 1523 & 1531 & 1543 & 1549 & 1553 & 1559 & 1567 & 1571 & 1579 & 1583 \\
\hline 1597 & 1601 & 1607 & 1609 & 1613 & 1619 & 1621 & 1627 & 1637 & 1657 \\
\hline 1663 & 1667 & 1669 & 1693 & 1697 & 1699 & 1709 & 1721 & 1723 & 1733 \\
\hline 1741 & 1747 & 1753 & 1759 & 1777 & 1783 & 1787 & 1789 & 1801 & 1811 \\
\hline 1823 & 1831 & 1847 & 1861 & 1867 & 1871 & 1873 & 1877 & 1879 & 1889 \\
\hline
\end{tabular}




\begin{tabular}{|c|c|c|c|c|c|c|c|c|c|}
\hline 95783 & 95789 & 95791 & 95801 & 95803 & 95813 & 95819 & 95857 & 95869 & 95873 \\
\hline 95881 & 95891 & 95911 & 95917 & 95923 & 95929 & 95947 & 95957 & 95959 & 95971 \\
\hline 95987 & 95989 & 96001 & 96013 & 96017 & 96043 & 96053 & 96059 & 96079 & 96097 \\
\hline 96137 & 96149 & 96157 & 96167 & 96179 & 96181 & 96199 & 96211 & 96221 & 96223 \\
\hline 96233 & 96259 & 96263 & 96269 & 96281 & 96289 & 96293 & 96323 & 96329 & 96331 \\
\hline 96337 & 96353 & 96377 & 96401 & 96419 & 96431 & 96443 & 96451 & 96457 & 96461 \\
\hline 96469 & 96479 & 96487 & 96493 & 96497 & 96517 & 96527 & 96553 & 96557 & 96581 \\
\hline 96587 & 96589 & 96601 & 96643 & 96661 & 96667 & 96671 & 96697 & 96703 & 96731 \\
\hline 96737 & 96739 & 96749 & 96757 & 96763 & 96769 & 96779 & 96787 & 96797 & 96799 \\
\hline 96821 & 96823 & 96827 & 96847 & 96851 & 96857 & 96893 & 96907 & 96911 & 96931 \\
\hline 96953 & 96959 & 96973 & 96979 & 96989 & 96997 & 97001 & 97003 & 97007 & 97021 \\
\hline 97039 & 97073 & 97081 & 97103 & 97117 & 97127 & 97151 & 97157 & 97159 & 97169 \\
\hline 97171 & 97177 & 97187 & 97213 & 97231 & 97241 & 97259 & 97283 & 97301 & 97303 \\
\hline 97327 & 97367 & 97369 & 97373 & 97379 & 97381 & 97387 & 97397 & 97423 & 97429 \\
\hline 97441 & 97453 & 97459 & 97463 & 97499 & 97501 & 97511 & 97523 & 97547 & 97549 \\
\hline 97553 & 97561 & 97571 & 97577 & 97579 & 97583 & 97607 & 97609 & 97613 & 97649 \\
\hline 97651 & 97673 & 97687 & 97711 & 97729 & 97771 & 97777 & 97787 & 97789 & 97813 \\
\hline 97829 & 97841 & 97843 & 97847 & 97849 & 97859 & 97861 & 97871 & 97879 & 97883 \\
\hline 97919 & 97927 & 97931 & 97943 & 97961 & 97967 & 97973 & 97987 & 98009 & 98011 \\
\hline 98017 & 98041 & 98047 & 98057 & 98081 & 98101 & 98123 & 98129 & 98143 & 98179 \\
\hline 98207 & 98213 & 98221 & 98227 & 98251 & 98257 & 98269 & 98297 & 98299 & 98317 \\
\hline 98321 & 98323 & 98327 & 98347 & 98369 & 98377 & 98387 & 98389 & 98407 & 98411 \\
\hline 98419 & 98429 & 98443 & 98453 & 98459 & 98467 & 98473 & 98479 & 98491 & 98507 \\
\hline 98519 & 98533 & 98543 & 98561 & 98563 & 98573 & 98597 & 98621 & 98627 & 98639 \\
\hline 98641 & 98663 & 98669 & 98689 & 98711 & 98713 & 98717 & 98729 & 98731 & 98737 \\
\hline 98773 & 98779 & 98801 & 98807 & 98809 & 98837 & 98849 & 98867 & 98869 & 98873 \\
\hline 98887 & 98893 & 98897 & 98899 & 98909 & 98911 & 98927 & 98929 & 98939 & 98947 \\
\hline 98953 & 98963 & 98981 & 98993 & 98999 & 99013 & 99017 & 99023 & 99041 & 99053 \\
\hline 99079 & 99083 & 99089 & 99103 & 99109 & 99119 & 99131 & 99133 & 99137 & 99139 \\
\hline 99149 & 99173 & 99181 & 99191 & 99223 & 99233 & 99241 & 99251 & 99257 & 99259 \\
\hline 99277 & 99289 & 99317 & 99347 & 99349 & 99367 & 99371 & 99377 & 99391 & 99397 \\
\hline 99401 & 99409 & 99431 & 99439 & 99469 & 99487 & 99497 & 99523 & 99527 & 99529 \\
\hline 99551 & 99559 & 99563 & 99571 & 99577 & 99581 & 99607 & 99611 & 99623 & 99643 \\
\hline 99661 & 99667 & 99679 & 99689 & 99707 & 99709 & 99713 & 99719 & 99721 & 99733 \\
\hline 99761 & 99767 & 99787 & 99793 & 99809 & 99817 & 99823 & 99829 & 99833 & 99839 \\
\hline 99859 & 99871 & 99877 & 99881 & 99901 & 99907 & 99923 & 99929 & 99961 & 99971 \\
\hline 99989 & 99991 & 100003 & 100019 & 100043 & 100049 & 100057 & 100069 & 100103 & 100109 \\
\hline & & & & & & & & & \\
\hline
\end{tabular}




\begin{tabular}{|c|c|c|c|c|}
\hline 888888888888811 & 888888888888859 & 888888888888883 & 888888888888907 & 888888888888953 \\
\hline 888888888888961 & 888888888888983 & 888888888888997 & 888888888889049 & 888888888889051 \\
\hline 888888888889087 & 888888888889091 & 888888888889097 & 888888888889187 & 888888888889189 \\
\hline 888888888889193 & 888888888889213 & 888888888889217 & 888888888889327 & 888888888889387 \\
\hline 888888888889471 & 888888888889499 & 888888888889537 & 888888888889559 & 888888888889583 \\
\hline 888888888889607 & 888888888889633 & 888888888889691 & 888888888889693 & 888888888889723 \\
\hline 888888888889751 & 888888888889871 & 888888888889873 & 888888888889879 & 888888888889891 \\
\hline 888888888889909 & 888888888889933 & 888888888889993 & 888888888890071 & 888888888890087 \\
\hline 888888888890147 & 888888888890209 & 888888888890473 & 888888888890521 & 888888888890537 \\
\hline 888888888890557 & 888888888890591 & 888888888890659 & 888888888890663 & 888888888890747 \\
\hline
\end{tabular}

My conjecture is that this method gives you all prime numbers without end.

A method giving every prime number without end

The formula above is a convenient way to find all the primes because you do not use so many operations. Even when you go from 888888888889723 to the next prime number 888888888889993 you just need fifteen 18 -adds. A minor problem is that you cannot follow one prime number to the next. But this is possible with the method I will now present.

This method also proceeds from the prime numbers $5,7,11,13,17$ and 19. Every prime number ends with the figures 1, 3, 7 or 9 . A number ending with 5 can never be a prime (except from 5). But every number ending with 5 , and derived from the primes 5, 7, 11, 13, 17 and 19, contains all prime numbers in relevant order, one by one, if you divide the original 5 -number with 5 . This does not occur every time, but quite often.

This is how the first 5 -numbers according to above are formed.

\begin{tabular}{|c|c||c|c|c|c|}
\hline 7 & 17 & 19 & 11 & 13 & 5 \\
\hline 25 & 35 & 55 & 65 & 85 & 95 \\
\hline
\end{tabular}

Let us look at the number 13 as an example. $13+18+18+18+18=85$ which divided with 5 gives the prime number 17, while for instance 175 divided with 5 gives the composite number $35(5 \times 7)$. This shows that every division with 5 does not give a prime. You should even notice that a 5 -number not connected with the starting primes always starts with both 3 and 5 which make them more complicated to work with. We therefore build a 5 -series derived from the starting primes and then find that these 5-numbers appear when 10 and 20 alternately are added to the previous 5 -number. Beneath the 48 first 5 -numbers are presented stack-wise.

\begin{tabular}{|l|l|l|l|l|l|l|l|}
\hline 25 & 115 & 205 & 295 & 385 & 475 & 565 & 655 \\
\hline 35 & 125 & 215 & 305 & 395 & 485 & 575 & 665 \\
\hline 55 & 145 & 235 & 325 & 415 & 505 & 595 & 685 \\
\hline 65 & 155 & 245 & 335 & 425 & 515 & 605 & 695 \\
\hline 85 & 175 & 265 & 355 & 445 & 535 & 625 & 715 \\
\hline 95 & 185 & 275 & 365 & 455 & 545 & 635 & 725 \\
\hline
\end{tabular}


To find every prime number, using a division with number 5 , you need to sort out all composite quotients. The numbers starting with the prime number 5 show themselves in a quite simple pattern and therefor are easy to find. The next composite number (after the 5-division) starting with, or containing, the prime number 7 starts with 7 squared, that is $5 \times 7^{2}$. In this way you get the exact position in the 5 -series where the 7 -s start, and this goes for all the following primes. Observe, though, that every analysis concerning every single 5 -number using this method starts with a 5-division.

You find that the pattern in which the 5-s, the 7-s, the 11-s and so on consists of one short and one long sequence. The short sequence is the investigated prime multiplied with 10 and the long sequence the prime multiplied with 20. It depends on the development of the 5-series if the investigated prime series starts with a short or a long sequence. The pattern in figures is shown beneath. The number beneath every prime is where to find the starting point, that is the prime number squared multiplied with 5 . The two numbers on the right show the short and the long sequence.

\begin{tabular}{|c|c|c|}
\hline 5 & 50 & 100 \\
\hline 125 & & \\
\hline 7 & 70 & 140 \\
\hline 245 & & \\
\hline 11 & 110 & 220 \\
\hline 605 & & \\
\hline 13 & 130 & 260 \\
\hline 845 & & \\
\hline 17 & 170 & 340 \\
\hline 1445 & & \\
\hline 19 & 190 & 380 \\
\hline 1805 & & \\
\hline 23 & 230 & 460 \\
\hline 2645 & & \\
\hline 29 & 290 & 580 \\
\hline 4205 & & \\
\hline 31 & 310 & 620 \\
\hline 4805 & & \\
\hline
\end{tabular}

When the 5-series is built and then every composite number is sorted out several gaps remain. These gaps, divided with 5, give every prime number in order. Then you find, for instance, why certain primes are twin primes and why there is a larger gap between certain prime numbers. The way the prime numbers show up is determined by how the composite 5 -s are positioned. The entire procedure is shown below. The prime numbers are yellow marked, and the starting position for every new prime (this prime squared $\times 5$ ) is blue marked. The horizontal lines show the six starting primes $7,17,19,11,13$ and 5. 


\begin{tabular}{|c|c|c|c|c|c|c|c|c|c|}
\hline 25 & 5 & 445 & 89 & 865 & 173 & 1285 & 257 & 1705 & $11 \times 31$ \\
\hline 35 & 7 & 455 & $7 \times 13$ & 875 & $5 \times 5 \times 7$ & 1295 & $7 \times 37$ & 1715 & $7 \times 7 \times 7$ \\
\hline 55 & 11 & 475 & $5 \times 19$ & 895 & 179 & 1315 & 263 & 1735 & 347 \\
\hline 65 & 13 & 485 & 97 & 905 & 181 & 1325 & $5 \times 53$ & 1745 & 349 \\
\hline 85 & 17 & 505 & 101 & 925 & $5 \times 37$ & 1345 & 269 & 1765 & 353 \\
\hline 95 & 19 & 515 & 103 & 935 & $11 \times 17$ & 1355 & 271 & 1775 & $5 \times 71$ \\
\hline 115 & 23 & 535 & 107 & 955 & 191 & 1375 & $5 \times 5 \times 11$ & 1795 & 359 \\
\hline 125 & $5 \times 5$ & 545 & 109 & 965 & 193 & 1385 & 277 & 1805 & $19 \times 19$ \\
\hline 145 & 29 & 565 & 113 & 985 & 197 & 1405 & 281 & 1825 & $5 \times 73$ \\
\hline 155 & 31 & 575 & $5 \times 23$ & 995 & 199 & 1415 & 283 & 1835 & 367 \\
\hline 175 & $5 \times 7$ & 595 & $7 \times 17$ & 1015 & $7 \times 29$ & 1435 & $7 \times 41$ & 1855 & $7 \times 53$ \\
\hline 185 & 37 & 605 & $11 \times 11$ & 1025 & $5 \times 41$ & 1445 & $17 \times 17$ & 1865 & 373 \\
\hline 205 & 41 & 625 & $5 \times 5 \times 5$ & 1045 & $11 \times 19$ & 1465 & 293 & 1885 & $13 \times 29$ \\
\hline 215 & 43 & 635 & 127 & 1055 & 211 & 1475 & $5 \times 59$ & 1895 & 379 \\
\hline 235 & 47 & 655 & 131 & 1075 & $5 \times 43$ & 1495 & $13 \times 23$ & 1915 & 383 \\
\hline 245 & $7 \times 7$ & 665 & $7 \times 19$ & 1085 & $7 \times 31$ & 1505 & $7 \times 43$ & 1925 & $5 \times 7 \times 11$ \\
\hline 265 & 53 & 685 & 137 & 1105 & $13 \times 17$ & 1525 & $5 \times 61$ & 1945 & 389 \\
\hline 275 & $5 \times 11$ & 695 & 139 & 1115 & 223 & 1535 & 307 & 1955 & $17 \times 23$ \\
\hline 295 & 59 & 715 & $11 \times 13$ & 1135 & 227 & 1555 & 311 & 1975 & $5 \times 79$ \\
\hline 305 & 61 & 725 & $5 \times 29$ & 1145 & 229 & 1565 & 313 & 1985 & 397 \\
\hline 325 & $5 \times 13$ & 745 & 149 & 1165 & 233 & 1585 & 317 & 2005 & 401 \\
\hline 335 & 67 & 755 & 151 & 1175 & $5 \times 47$ & 1595 & $11 \times 29$ & 2015 & $13 \times 31$ \\
\hline 355 & 71 & 775 & $5 \times 23$ & 1195 & 239 & 1615 & $17 \times 19$ & 2035 & $11 \times 37$ \\
\hline 365 & 73 & 785 & 157 & 1205 & 241 & 1625 & $5 \times 5 \times 13$ & 2045 & 409 \\
\hline 385 & $7 \times 11$ & 805 & $7 \times 23$ & 1225 & $5 \times 7 \times 7$ & 1645 & $7 \times 47$ & 2065 & $7 \times 59$ \\
\hline 395 & 79 & 815 & 163 & 1235 & $13 \times 19$ & 1655 & 331 & 2075 & $5 \times 83$ \\
\hline 415 & 83 & 835 & 167 & 1255 & 251 & 1675 & 567 & 2095 & 419 \\
\hline 425 & $5 \times 17$ & 845 & $13 \times 13$ & 1265 & $11 \times 23$ & 1685 & 337 & 2105 & 421 \\
\hline
\end{tabular}




\begin{tabular}{|c|c|c|c|c|c|c|c|c|c|}
\hline 2125 & $5 \times 5 \times 17$ & 2545 & 509 & 2965 & 593 & 3385 & 677 & 3805 & 761 \\
\hline 2135 & $7 \times 61$ & 2555 & $7 \times 73$ & 2975 & $5 \times 7 \times 17$ & 3395 & $7 \times 97$ & 3815 & $7 \times 109$ \\
\hline 2155 & 431 & 2575 & $5 \times 103$ & 2995 & 599 & 3415 & 683 & 3835 & $13 \times 59$ \\
\hline 2165 & 433 & 2585 & $11 \times 47$ & 3005 & 601 & 3425 & $5 \times 137$ & 3845 & 769 \\
\hline 2185 & $19 \times 23$ & 2605 & 521 & 3025 & $5 \times 11 \times 11$ & 3445 & $13 \times 53$ & 3865 & 773 \\
\hline 2195 & 439 & 2615 & 523 & 3035 & 607 & 3455 & 691 & 3875 & $5 \times 5 \times 31$ \\
\hline 2215 & 443 & 2635 & $17 \times 31$ & 3055 & $13 \times 47$ & 3475 & $5 \times 139$ & 3895 & $19 \times 41$ \\
\hline 2225 & $5 \times 89$ & 2645 & $23 \times 23$ & 3065 & 613 & 3485 & $17 \times 41$ & 3905 & $11 \times 71$ \\
\hline 2245 & 449 & 2665 & $13 \times 41$ & 3085 & 617 & 3505 & 701 & 3925 & $5 \times 157$ \\
\hline 2255 & $11 \times 41$ & 2675 & $5 \times 107$ & 3095 & 619 & 3515 & $19 \times 37$ & 3935 & 787 \\
\hline 2275 & $5 \times 7 \times 13$ & 2695 & $7 \times 7 \times 11$ & 3115 & $7 \times 89$ & 3535 & $7 \times 101$ & 3955 & $7 \times 113$ \\
\hline 2285 & 457 & 2705 & 541 & 3125 & $5 \times 5 \times 5 \times 5$ & 3545 & 709 & 3965 & $13 \times 61$ \\
\hline 2305 & 461 & 2725 & $5 \times 109$ & 3145 & $17 \times 37$ & 3565 & $23 \times 31$ & 3985 & 797 \\
\hline 2315 & 463 & 2735 & 547 & 3155 & 631 & 3575 & $5 \times 11 \times 13$ & 3995 & $17 \times 47$ \\
\hline 2335 & 467 & 2755 & $19 \times 29$ & 3175 & $5 \times 127$ & 3595 & 719 & 4015 & $11 \times 73$ \\
\hline 2345 & $7 \times 67$ & 2765 & $7 \times 79$ & 3185 & $7 \times 7 \times 13$ & 3605 & $7 \times 103$ & 4025 & $5 \times 7 \times 23$ \\
\hline 2365 & $11 \times 43$ & 2785 & 557 & 3205 & 641 & 3625 & $5 \times 5 \times 29$ & 4045 & 809 \\
\hline 2375 & $5 \times 5 \times 19$ & 2795 & $13 \times 43$ & 3215 & 643 & 3635 & 727 & 4055 & 811 \\
\hline 2395 & 479 & 2815 & 563 & 3235 & 647 & 3655 & $17 \times 43$ & 4075 & $5 \times 163$ \\
\hline 2405 & $13 \times 37$ & 2825 & $5 \times 113$ & 3245 & $11 \times 59$ & 3665 & 733 & 4085 & $19 \times 43$ \\
\hline 2425 & $5 \times 97$ & 2845 & 569 & 3265 & 653 & 3685 & $11 \times 67$ & 4105 & 821 \\
\hline 2435 & 487 & 2855 & 571 & 3275 & $5 \times 131$ & 3695 & 739 & 4115 & 823 \\
\hline 2455 & 491 & 2875 & $5 \times 5 \times 23$ & 3295 & 659 & 3715 & 743 & 4135 & 827 \\
\hline 2465 & $17 \times 29$ & 2885 & 577 & 3305 & 661 & 3725 & $5 \times 149$ & 4145 & 829 \\
\hline 2485 & $7 \times 71$ & 2905 & $7 \times 83$ & 3325 & $5 \times 7 \times 19$ & 3745 & $7 \times 107$ & 4165 & $7 \times 7 \times 17$ \\
\hline 2495 & 499 & 2915 & $11 \times 53$ & 3335 & $23 \times 29$ & 3755 & 751 & 4175 & $5 \times 167$ \\
\hline 2515 & 503 & 2935 & 587 & 3355 & $11 \times 61$ & 3775 & $5 \times 151$ & 4195 & 839 \\
\hline 2525 & $5 \times 101$ & 2945 & $19 \times 31$ & 3365 & 673 & 3785 & 757 & 4205 & $29 \times 29$ \\
\hline
\end{tabular}




\begin{tabular}{|c|c|c|c|c|c|c|c|c|c|}
\hline 4225 & $5 \times 13 \times 13$ & 4645 & 929 & 5065 & 1013 & 5485 & 1097 & 5905 & 1181 \\
\hline 4235 & $7 \times 11 \times 11$ & 4655 & $7 \times 7 \times 19$ & 5075 & $5 \times 7 \times 29$ & 5495 & $7 \times 157$ & 5915 & $7 \times 13 \times 13$ \\
\hline 4255 & $23 \times 37$ & 4675 & $5 \times 11 \times 17$ & 5095 & 1019 & 5515 & 1103 & 5935 & 1187 \\
\hline 4265 & 853 & 4685 & 937 & 5105 & 1021 & 5525 & $5 \times 13 \times 17$ & 5945 & $29 \times 41$ \\
\hline 4285 & 857 & 4705 & 941 & 5125 & $5 \times 5 \times 41$ & 5545 & 1109 & 5965 & 1193 \\
\hline 4295 & 859 & 4715 & $23 \times 41$ & 5135 & $13 \times 79$ & 5555 & $11 \times 101$ & 5975 & $5 \times 239$ \\
\hline 4315 & 863 & 4735 & 947 & 5155 & 1031 & 5575 & $5 \times 223$ & 5995 & $11 \times 109$ \\
\hline 4325 & $5 \times 173$ & 4745 & $13 \times 73$ & 5165 & 1033 & 5585 & 1117 & 6005 & 1201 \\
\hline 4345 & $11 \times 79$ & 4765 & 953 & 5185 & $17 \times 61$ & 5605 & $19 \times 59$ & 6025 & $5 \times 241$ \\
\hline 4355 & $13 \times 67$ & 4775 & $5 \times 191$ & 5195 & 1039 & 5615 & 1123 & 6035 & $17 \times 71$ \\
\hline 4375 & $5 \times 5 \times 5 \times 7$ & 4795 & $7 \times 137$ & 5215 & $7 \times 149$ & 5635 & $7 \times 7 \times 23$ & 6055 & $7 \times 173$ \\
\hline 4385 & 877 & 4805 & $31 \times 31$ & 5225 & $5 \times 11 \times 19$ & 5645 & 1129 & 6065 & 1213 \\
\hline 4405 & 881 & 4825 & $5 \times 193$ & 5245 & 1049 & 5665 & $11 \times 103$ & 6085 & 1217 \\
\hline 4415 & 883 & 4835 & 967 & 5255 & 1051 & 5675 & $5 \times 227$ & 6095 & $23 \times 53$ \\
\hline 4435 & 887 & 4855 & 971 & 5275 & $5 \times 211$ & 5695 & $17 \times 67$ & 6115 & 1223 \\
\hline 4445 & $7 \times 127$ & 4865 & $7 \times 139$ & 5285 & $7 \times 151$ & 5705 & $7 \times 163$ & 6125 & $5 \times 5 \times 7 \times 7$ \\
\hline 4465 & $19 \times 47$ & 4885 & 977 & 5305 & 1061 & 5725 & $5 \times 229$ & 6145 & 1229 \\
\hline 4475 & $5 \times 179$ & 4895 & $11 \times 89$ & 5315 & 1063 & 5735 & $31 \times 37$ & 6155 & 1231 \\
\hline 4495 & $29 \times 31$ & 4915 & 983 & 5335 & $11 \times 97$ & 5755 & 1151 & 6175 & $5 \times 13 \times 19$ \\
\hline 4505 & $17 \times 53$ & 4925 & $5 \times 197$ & 5345 & 1069 & 5765 & 1153 & 6185 & 1237 \\
\hline 4525 & $5 \times 181$ & 4945 & $23 \times 43$ & 5365 & $29 \times 37$ & 5785 & $13 \times 89$ & 6205 & $17 \times 73$ \\
\hline 4535 & 907 & 4955 & 991 & 5375 & $5 \times 5 \times 43$ & 5795 & $19 \times 61$ & 6215 & $11 \times 113$ \\
\hline 4555 & 911 & 4975 & $5 \times 199$ & 5395 & $13 \times 83$ & 5815 & 1163 & 6235 & $29 \times 43$ \\
\hline 4565 & $11 \times 83$ & 4985 & 997 & 5405 & $23 \times 47$ & 5825 & $5 \times 233$ & 6245 & 1249 \\
\hline 4585 & $7 \times 131$ & 5005 & $7 \times 11 \times 13$ & 5425 & $5 \times 7 \times 31$ & 5845 & $7 \times 167$ & 6265 & $7 \times 179$ \\
\hline 4595 & 919 & 5015 & 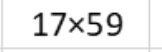 & 5435 & 1087 & 5855 & 1171 & 6275 & $5 \times 251$ \\
\hline 4615 & $13 \times 71$ & 5035 & $19 \times 53$ & 5455 & 1091 & 5875 & $5 \times 5 \times 47$ & 6295 & 1259 \\
\hline 4625 & $5 \times 5 \times 37$ & 5045 & 1009 & 5465 & 1093 & 5885 & $11 \times 107$ & 6305 & $13 \times 97$ \\
\hline
\end{tabular}

The survey above shows how and why the prime numbers appear the way they do. You may even notice that the composite numbers, after the 5-division, show a pattern where the prime number series appear in many different variations. My conjecture is that this method gives you all prime numbers without end.

\section{Summary}

My investigation shows that there is a regularity even by the prime numbers. This structure is obvious when a prime square is created. The squared prime numbers.

\section{Connections in a prime square}

A prime square (or origin square) is defined as a square consisting of as many boxes as the origin prime squared. This prime settle every side of the square. So, for example, the origin square 17 has got four sides with 17 boxes along every side. The prime numbers in each of the 289 boxes are filled with primes when a prime number occur in the number series $(1,2,3,4,5,6,7,8,9$ and so on) and then is noted in that very box.

If a box is occupied in the origin square $A$ this prime number could be transferred to the corresponding box in a second square $B$, and thereafter the counting and noting continue in the first square A. Eventually we get two filled prime squares. Analyzing these squares, you leave out the right vertical line, representing only the origin prime number.

When a square is filled with primes you subdivide it into four corner squares, as big as possible, denoted a, b, $c$ and $d$ clockwise. You also get a center line between the left and right vertical sides.

Irrespective of what kind of constellation you activate this is what you find: 
1. Every constellation in the corner square a and/or $d$ added to a corresponding constellation in the corner square $b$ and/or $c$ is evenly divisible with the origin prime.

2. Every constellation in the corner square $a$ and/or $b$ added to a corresponding constellation in the corner square $d$ and/or $c$ is not evenly divisible with the origin prime.

3. Every reflecting constellation inside two of the opposed diagonal corner squares, possibly summarized with any optional reflecting constellation inside the two other diagonal corner squares, is evenly divisible with the origin prime squared. You may even add a reflection inside the center line and get this result.

My Conjecture 1 is that this applies to every prime square without end.

\section{A formula giving all prime numbers endless}

In the second prime square the prime numbers are always higher than in the first square if you compare a specific box. There is a mathematic connection between the prime numbers in the first and second square. This connection appears when you square and double the origin prime and thereafter add this number to the prime you investigate. A new higher prime is found after $n$ additions.

You start with the lowest applicable prime number 3 and its square $3^{2}$. Double it and you get 18 . We add 18 to the six next prime numbers 5, 7, 11, 13, 17 and 19 in any order. After a few adds you get a prime and after another few adds you get another higher one. In this way you continue as long as you want to. The primes are creating themselves.

\section{A formula giving all prime numbers is:}

$$
\begin{aligned}
& 5+18 \times n,+18 \times n,+18 \times n \ldots \text { without end } \\
& 7+18 \times n,+18 \times n,+18 \times n \ldots \text { without end } \\
& 11+18 \times n,+18 \times n,+18 \times n \ldots \text { without end } \\
& 13+18 \times n,+18 \times n,+18 \times n \ldots \text { without end } \\
& 17+18 \times n,+18 \times n,+18 \times n \ldots \text { without end } \\
& 19+18 \times n,+18 \times n,+18 \times n \ldots \text { without end }
\end{aligned}
$$

The letter $n$ in the formula stands for how many 18 -adds you must do until the next prime is found.

My Conjecture 2 is that you find every prime number by adding 18 to the primes 5, 7, 11, 13, 17 and 19 one by one endless.

\section{A method giving all prime numbers endless}

There is still a possibility to even more precise get all prime numbers. You start a 5 -number series derived from the start primes $7,17,19,11,13$ and 5 in that very order. Factorized these number always begin with number 5 . When each of these numbers are divided with 5 the quotient is either a prime number or a composite number containing two or some more prime numbers in the nearby. By sorting out all the composite quotients you get all the prime numbers endless and in order.

Every composite quotient starts with a prime from 5 and up, squared. Thereafter the quotients starting with that prime show up periodically according to a pattern of short and long sequences. The position for each new prime beginning the composite quotient is this prime squared and multiplied with 5 . Thereafter the short sequence is this prime multiplied with 10, while the long sequence is this prime multiplied with 20 .

When all the composite quotients are deleted there are left several 5 -numbers which divided with 5 give all prime numbers, and you even see clearly the distance between the prime numbers which for instance explain why the prime twins occur as they do.

My Conjecture 3 is that this is an exact method giving all prime numbers endless and in order. 